\title{
DUAL MODULES OVER A VALUATION RING. I
}

\section{IRVING KAPLANSKY ${ }^{1}$}

1. Introduction. The present investigation is inspired by a series of papers in the literature, beginning with Prüfer $[8],{ }^{2}$ and continuing with Pietrkowski $[6 ; 7]$, Krull $[2 ; 3]$, Vilenkin $[10 ; 11]$, and Schöneborn [9]. While the formulation varies somewhat from paper to paper, the fundamental object of study in all of them is a linearly compact module over the p-adic integers, in the sense of the following definition.

Definition. Let $R$ be a topological ring. By a linearly compact $R$-module we mean a topological $R$-module $M$ (multiplication between $R$ and $M$ jointly continuous) satisfying the following two conditions:

(a) $M$ has a set of submodule neighborhoods of 0 ,

(b) $M$ has the "compactness" property for cosets of closed submodules; that is, if a set of congruences

$$
x \equiv a_{i}\left(\bmod S_{i}\right), \quad S_{i} \text { a closed submodule of } M,
$$

is such that every finite subset has a solution in $M$, then the entire set has a solution in $M$.

If $R$ is a (discrete) field this coincides with the definition given by Lefschetz [4]. The basic properties of linearly compact modules have been worked out by Zelinsky [12]. In this paper we are concerned with the case where $R$ is the ring of $p$-adic integers, or more generally a complete discrete valuation ring, and we shall develop a theory of duality.

In [3] Krull observed that a compact totally disconnected primary ${ }^{3}$ abelian group $G$ is an example of a linearly compact module over the $p$-adic integers. Now Pontrjagin's duality theory reduces the study of $G$ to that of its character group $G^{*}$, a discrete primary group about which an immense amount is known. The author's contribution in this paper is the observation that the most general linearly compact module over the $p$-adic integers is similarly dual to an (equally general) discrete module. The duality has to be taken, not relative to the reals mod one, but to its primary subgroup (Prüfer's group of type $\left.p^{\infty}\right)$.

Presented to the Society, September 5, 1952; received by the editors June 1, 1952.

${ }^{1}$ This research was supported in part by the Office of Naval Research.

2 Numbers in brackets refer to the bibliography at the end of the paper.

3 "Primary" means that $p^{n} x \rightarrow 0$ for every $x$. 
In $\$ 5$ we indicate how this duality clarifies the work of the authors cited above.

2. Abstract dual modules. In proving certain basic results it is enlightening to begin, not with topological modules, but with a purely algebraic formulation after the model of Mackey [5].

Let $R$ be a complete discrete valuation ring, ${ }^{4} K$ its quotient field. We shall denote by $R_{0}$ the $R$-module $K / R$. We say that the $R$-modules $M$ and $N$ are dual (or that $M$ is paired to $N$ ) if we are given a bilinear inner product $(x, y)$ defined for $x$ in $M, y$ in $N$ and taking values in $R_{0}$, which is nondegenerate in the sense that no nonzero element of $M$ (resp. $N$ ) annihilates all of $N$ (resp. $M$ ).

Let $S$ be a submodule of $M$. We define its annihilator $S^{\prime}$ to be the set of all $y$ in $N$ with $(S, y)=0$. We call $S$ closed if $S=S^{\prime \prime}$. There is a natural way of construing $S$ and $N / S^{\prime}$ to be dual modules; if $S$ is closed we can do the same with $M / S$ and $S^{\prime}$.

We leave to the reader the proof of the following lemma.

Leмма 1. Let $M$ and $N$ be dual modules, $S$ a closed submodule of $M$, and $T$ a submodule of $M$ containing $S$. Then $T$ is closed if and only if $T / S$ is closed in $M / S$ (the latter being paired to $S^{\prime}$ ).

By the full dual of an $R$-module $M$ we mean the module of all homomorphisms of $M$ into $R_{0}$. We shall see in Lemma 4 that $M$ and its full dual form a pair of dual modules. Note that if $M$ and $N$ are dual modules, then $N$ is a submodule of the full dual of $M$. [5].

The following is essentially a generalization of Theorem III-1 of

LEмma 2. Let $M$ and $N$ be dual modules, and let $S$ be a submodule of $M$ of finite rank. ${ }^{5}$ Then $S$ is closed. Also, $S$ induces the full dual of $N / S^{\prime}$.

Proof. By a finite number of applications of Lemma 1 we reduce the problem of proving that $S$ is closed to the case where $S$ is of rank one. Then $S$ is known to be isomorphic to one of the four modules $R / p^{i}, R, R_{0}$, or $K$. Since $K$ may be treated in the two steps $R$, and $K / R=R_{0}$, we need actually only consider the first three possibilities. The fact that $S$ is closed now follows from the following easily verified remark: if $T$ is isomorphic to $R$ (resp. $R_{0}$ or $R / p^{i}$ ), then the full dual of $T$ is isomorphic to $R_{0}$ (resp. $R$ or $R / p^{i}$ ), and

\footnotetext{
That is, an integral domain with a unique nonzero prime ideal $(p)$, complete in the topology given by the powers of $p$.

- An $R$-module is of rank $n$ if every finitely generated submodule can be generated by $\boldsymbol{n}$ or fewer elements, and $\boldsymbol{n}$ is the smallest such integer. It is known that an $R$ module of finite rank is the direct sum of modules of rank one.
} 
moreover $T$ cannot be paired to any proper submodule of its full dual.

In proving the last part of Lemma 2, we can assume without loss of generality that $S$ is all of $M$. In other words, $M$ is now of finite rank and we wish to show that it coincides with the full dual (say $M_{1}$ ) of $N$. Now $M_{1}$ and $N$ are dually paired (this is clear even without Lemma 4, for no nonzero element of $N$ can annihilate $M$, let alone $\left.M_{1}\right)$. Relative to the pair $M_{1}, N$ we have that $M^{\prime}=0, M^{\prime \prime}=M_{1}$. But we showed above that $M$ is closed. Hence $M=M_{1}$, as desired.

Since we now know that for modules of finite rank all submodules will be closed, and since annihilation sets up a one-one order-inverting correspondence between the closed submodules, the next lemma is clear.

Lemma 3. Let $M$ and $N$ be dual modules. Then $M$ satisfies the descending chain condition if and only if $N$ satisfies the ascending chain condition.

3. The dual of a topological module. Let $M$ be a topological $R$ module, where $R$ (as always) is a complete discrete valuation ring. We define the dual $M^{*}$ to be the module of all continuous homomorphisms from $M$ into $R_{0}$, where $R_{0}$ has been awarded the discrete topology. It is evident that a homomorphism from $M$ into $R_{0}$ is continuous if and only if its kernel is open.

It is not within the scope of this paper to discuss topologies for $M^{*}$.

LEMмA 4. Let $M$ be a topological $R$-module with submodule neighborhoods of 0 , and $M^{*}$ its dual. Then $M$ and $M^{*}$ form a pair of dual modules, that is, no nonzero element of $M$ is annihilated by all of $M^{*}$.

Proof. Let $x$ be a nonzero element in $M$. Select an open submodule $U$ of $M$, with $x$ not in $U$. Let $y$ denote the image of $x$ in $M / U$. We can define a nontrivial homomorphism $f$ of the cyclic submodule generated by $y$ into $R_{0}$ (for example, send $y$ into an element of order $p$ ). It is well known that $f$ can be extended to a homomorphism $g$ of all of $M / U$ into $R_{0}$. By lifting up $g$ to $M$ we obtain the desired continuous homomorphism not vanishing at $x$.

LEMMA 5. Let $M$ be a topological $R$-module with submodule neighborhoods of 0 , and $S$ a (topologically) closed submodule. Then $S$ is also closed in the algebraic sense of being a double annihilator relative to the dual modules $M, M^{*}$.

Proof. The problem is to prove the existence of an element in $M^{*}$, vanishing on $S$, but not vanishing at a prescribed element not in $S$. But the quotient module $M / S$ again has submodule neighborhoods of 0 , and to it we apply Lemma 4. 
We remark that conversely an algebraically closed submodule is topologically closed, for it is the intersection of the kernels of continuous functionals.

4. Duality theorem. We are now ready to take up linearly compact modules, and we first settle the question of when they are discrete.

Leмма 6. A linearly compact $R$-module is discrete if and only if it satisfies the descending chain condition.

Proof. Suppose $M$ is both linearly compact and discrete. For any $x$ in $M$ we have, by the continuity of multiplication, that there exists a neighborhood $V$ of 0 in $R$ such that $V x=0$. This says that $x$ is annihilated by some power of $p$, that is, $M$ is a torsion module. Next, let $P$ be the submodule of $M$ annihilated by $p ; P$ is a closed submodule and hence is again linearly compact $^{6}$ (as well as discrete). Now $P$ may be regarded as a vector space over the field $R /(p)$; we borrow from the theory of linearly compact vector spaces the fact that $P$ is finite-dimensional. It is known that this (together with the fact that $M$ is a torsion module) implies that $M$ has the descending chain condition.

The converse is easy and valid over any topological ring.

To continue the analysis, we let $M^{*}$ denote the dual of the linearly compact module $M$. We decree that $M^{*}$ is to be discrete (although this point deserves further discussion). It is now possible to form the (full) dual $M^{* *}$ of $M^{*}$, and by Lemma $4, M$ is isomorphically embedded in $M^{* *}$.

THEOREM. Let $R$ be a complete discrete valuation ring and $M$ a linearly compact $R$-module. Then $M$ coincides with its second dual $M^{* *}$ both algebraically and topologically, where $M^{* *}$ is given the weak topology induced by $M^{*}$.

Proof. First we establish the algebraic isomorphism, that is, we take an element $z$ in $M^{* *}$ and show that $z$ lies in $M$. For any finitely generated submodule $S_{i}$ of $M^{*}$ we have that $z$ induces a homomorphism of $S_{i}$ into $R_{0}$. By Lemma 2 this homomorphism can also be induced by an element $b_{i}$ of $M / S_{i}^{\prime}$. Let $a_{i}$ be any element of $M$ mapping on $b_{i}$. We form the congruences

$$
x \equiv a_{i}\left(\bmod S_{i}^{\prime}\right)
$$

and observe that any finite number have a solution. By linear com-

- Any closed submodule or continuous image of a linearly compact module is linearly compact [12]. 
pactness there exists a solution $x$ of the entire set, and manifestly $z=x$.

To check the coincidence of the topologies we begin by observing that a neighborhood of 0 in the weak topology for $M$ is precisely the annihilator of a finitely generated submodule of $M^{*}$. But an open submodule of $M$, in the original topology, is exactly the same thing. For the annihilator of a finitely generated submodule of $M^{*}$ is open, being the intersection of a finite number of open kernels of functionals. Conversely, let $U$ be an open submodule (hence closed). By Lemma 5, $U$ is the annihilator of $U^{\prime}$. By Lemma 6, $M / U$ has the descending chain condition. By Lemma $3, U^{\prime}$ is finitely generated, since it is paired to $M / U$.

5. Further remarks. (i) As an example of the use of duality, let us look at two of the theorems in [3]. The first Hauptsatz on p. 36 asserts that a torsion-free linearly compact module over the $p$-adic integers is a (complete) direct sum of modules of rank one. It turns out that the hypothesis that $M$ is torsion-free translates into the statement that $M^{*}$ is divisible $\left(p M^{*}=M^{*}\right)$. Then one knows that $M^{*}$ is a direct sum of copies of $K$ or $R_{0}$. Dualizing, $M$ is a direct sum of copies of $K$ or $R$. (One notes that the countability hypothesis that runs through the work of Krull and Vilenkin is not needed here.)

The second Hauptsatz on p. 37 asserts more generally that a "korrekt verknottet" module is a complete direct sum of modules of rank one. Let $T$ be the closure of the torsion submodule of $M$; its annihilator $T^{\prime}$ is the set of elements of infinite height in $M^{*}$. Now the "korrekt verknottet" hypothesis splits into two parts, the first part saying that $M / T$ is torsion-free. This dualizes to $T^{\prime}$ being divisible, whence it is a direct summand of $M^{*}$, say $M^{*}=T^{\prime} \oplus W$. The second half of the hypothesis asserts that in $p^{\infty} T=\cap p^{n} T$ the torsion submodule is dense. If $X$ is the torsion submodule of $W$, the dual statement is that $W / X$ has no elements of infinite height. Now assume $W$ is countably generated; then a theorem of Prüfer tells us that $W / X$ is free. This makes $X$ a direct summand of $W$. Another theorem of Prüfer tells us that $X$ is a direct sum of cyclic modules. In summary: the second Hauptsatz translates into two theorems of Prüfer concerning discrete modules, both of them requiring countability.

(ii) The work of Vilenkin falls nicely into place. A "coseparable" group turns out to be an arbitrary module over the $p$-adic integers, equipped with the compact-open topology induced by its full dual. Similarly, the "weakly separable" groups are locally linearly compact modules. The reader may convince himself that the results in $[10$, Part II] and [11] thus become reasonably transparent. 
(iii) The theory of dual modules has a connection with locally compact abelian groups. One knows-cf. [1] - that the latter theory may be reduced largely to the primary case. For primary locally compact abelian groups the duality is officially taken relative to the reals mod one; but actually only the primary subgroup of the reals mod one shows up at all as values, as was observed by Krull in footnote 51 of [3]. In other words, locally compact primary abelian groups are a special case of dual modules.

(iv) There is an application to linear transformations. Let $V$ and $W$ be dual vector spaces with an inner product $(x, y)$ in a field $F$. Let $T$ be a linear transformation on $V$ which is locally nilpotent (every vector annihilated by some power of $T$ ); suppose it has an adjoint $T^{*}$ on $W$ which is also locally nilpotent. Let $R$ be the ring of all formal power series over $F$ in a variable $u$. We can make $V, W$ into dual $R$-modules by having $u$ act on $V, W$ as $T, T^{*}$ respectively and defining the inner product as

$$
[x, y]=(x, y) u^{-1}+(T x, y) u^{-2}+\cdots \quad(\bmod R) .
$$

The classification of $T$ is equivalent to the classification of these dual modules.

(v) The particular case of dual $R$-modules annihilated by $p$ coincides with Mackey's theory of dual vector spaces. With a slight exaggeration we may say that locally compact abelian groups and locally convex topological linear spaces have thus merged into a single subject.

It seems clear that dual modules merit systematic investigation, and we propose to do this in a subsequent paper.

\section{Bibliography}

1. J. Braconnier, Sur les groupes topologiques localement compacts, J. Math. Pures Appl. vol. 27 (1948) pp. 1-85.

2. W. Krull, Über separable, abgeschlossene abelsche Gruppen, J. Reine Angew. Math. vol. 182 (1940) pp. 235-241.

3. - Über separable, insbesondere kompakte separable Gruppen, J. Reine Angew. Math. vol. 184 (1942) pp. 19-48.

4. S. Lefschetz, Algebraic topology, Amer. Math. Soc. Colloquium Publications, vol. 27, New York, 1942.

5. G. W. Mackey, On infinite-dimensional linear spaces, Trans. Amer. Math. Soc. vol. 57 (1945) pp. 155-207.

6. S. Pietrkowski, Theorie der unendlichen abelschen Gruppen, Math. Ann. vol. 104 (1931) pp. 535-569.

7. - Untergruppen und Quotientengruppen unendlicher abelscher Gruppen, Math. Ann. vol. 105 (1931) pp. 666-671.

8. H. Prüfer, Theorie der abelschen Gruppen. II. Ideale Gruppen, Math. Zeit. vol. 22 (1925) pp. 222-249. 
9. H. Schöneborn, Über Linearformenmoduln unendlichen Ranges. I. Primäre, kompakte Linearformenmoduln, J. Reine Angew. Math. vol. 189 (1951) pp. 168-185.

10. N. Vilenkin, Direct decompositions of topological groups. I, II, Mat. Sbornik vol. 19 (1946) pp. 85-154, 311-340. Amer. Math. Soc. Translation no. 23.

11. - On the theory of weakly separable groups, Mat. Sbornik vol. 22 (1948) pp. 135-177.

12. D. Zelinsky, Linearly compact modules and rings, Amer. J. Math. vol. 75 (1953) pp. 79-90.

University of Chicago

\section{A PROOF OF HESSENBERG'S THEOREM}

\section{ARNO CRONHEIM}

About fifty years ago Hessenberg [1] discovered that in a projective plane the Pappus property implies the Desargues property. In all the proofs of this beautiful theorem [2-5] only the so-called general case is treated so that some of the points and lines constructed in the course of the proof may actually become indeterminate. In the following lines we offer a proof of this theorem which takes care of all possible cases. We show first how to reduce the discussion to two cases only. The first of these cases may be treated by Hessenberg's argument, but in the second one a different proof is needed.

We consider now a projective plane. Whenever the points $X, Y$, and $Z$ are collinear, then we write $(X, Y, Z)$; and the fact that they are not collinear we indicate by writing $\operatorname{non}(X, Y, Z)$. In order to prove the Desarguesian closure property for our projective plane it suffices to consider seven distinct points $A_{i}, B_{i}$, and $S$ such that the three lines $A_{i}+B_{i}$ are distinct and meet in $S$ and neither $\left(A_{1}, A_{2}, A_{3}\right)$ nor $\left(B_{1}, B_{2}, B_{3}\right)$. The following two properties of such a configuration are independent of the Pappus property.

(1) non $\left(A_{i}, B_{i}, B_{k}\right)$ and $\operatorname{non}\left(A_{i}, A_{k}, B_{k}\right)$ for $i \neq k$.

Proof. This is obvious since $S+A_{i}+B_{i} \neq S+A_{k}+B_{k}$ for $i \neq k$.

(2) If there does not exist a permutation $(i, j, k)$ of the numbers $(1,2,3)$ such that $\operatorname{non}\left(A_{i}, B_{j}, B_{k}\right)$ and $\operatorname{non}\left(B_{k}, A_{i}, A_{j}\right)$ simultaneously, then either $\left(A_{x}, B_{y}, B_{z}\right)$ for all permutations $(x, y, z)$ or $\left(B_{x}, A_{y}, A_{z}\right)$ for all permutations $(x, y, z)$.

Proof. Let us assume without loss of generality non $\left(B_{1}, A_{2}, A_{3}\right)$. It follows from the hypothesis of (2) that $\left(A_{2}, B_{1}, B_{3}\right)$ and $\left(A_{3}, B_{1}, B_{2}\right)$. $\left(B_{3}, A_{1}, A_{2}\right)$, together with $\left(A_{2}, B_{1}, B_{3}\right)$, would contradict

Received by the editors May 18, 1952. 